\title{
Transcranial magnetic stimulation can influence the selection of motor programmes
}

\author{
K Ammon, S C Gandevia
}

\begin{abstract}
Transcranial magnetic stimulation is becoming increasingly popular to study the rapidly conducting output from the motor cortex. Little is known about the effects of such stimuli on other aspects of cortical function. In the study single magnetic stimuli, subthreshold for movement, produced significant preference for selection of one hand in a forced-choice task. The hand preference depended upon the direction of the induced current. It occurred when the coil was positioned over frontal but not occipital cortex and was not mimicked by weak DC stimulation. Single magnetic stimuli which do not evoke movement can alter high-level motor planning.
\end{abstract}

This study was undertaken to determine whether single transcranial magnetic stimuli can alter the selection of a particular movement strategy. It is now well established that single electrical and magnetic stimuli can access the corticospinal output to many human motoneuron pools. ${ }^{1-4}$ This is believed to involve, in part, activation of pyramidal tract cells either directly, or trans-synaptically. ${ }^{5-7}$ Furthermore, the direction of the flow of induced current influences which cellular elements within the motor cortex are activated. Thus the threshold and latency of responses in the intrinsic muscles of the hand are significantly altered by whether, when viewed from above, the induced current flows in a clockwise or anticlockwise direction around the vertex. ${ }^{89}$ There has been less interest in whether the stimuli can alter other cortical functions. There is some evidence that they may evoke sensations of movement in non-naive subjects ${ }^{10}$ and that they may delay voluntary output from the motor cortex. ${ }^{11}$ Neither of these results implies that stimuli subthreshold for movement can alter cortical processing.

\section{Methods}

In the first of the studies, nine subjects received stimuli from a commercial magnetic coil (Novametrix). All subjects were righthanded according to a standard questionnaire. ${ }^{12}$ The subject was comfortably seated and the centre of the coil was positioned (tangentially) over $\mathrm{Fz}$, that is, anterior to the usual site for activation of upper limb muscles. ${ }^{8}$ To allow delivery of repeated trials under the same conditions the coil position was fixed about $5-10 \mathrm{~mm}$ above the scalp with an external frame. With the coil clamped, the usual stimulus intensity was about $50-80 \%$ of the maximal output.

Electromyographic studies confirmed that at the stimulus intensities used there were no responses in the distal muscles of the arm or leg. EMG responses and slight movements of the fingers were evoked by the stimuli during deliberate voluntary contractions. The subjects were instructed to relax and no evoked distal movements occurred during the studies. The stimuli produced a brief audible click, and slight contraction of scalp musculature due to activation of local intramuscular nerves. Following each stimulus the subject was required to extend either the left or right index finger within 2-5 s. The subject was asked to make this selection after delivery of the stimulus and not to select one side repeatedly. No further instructions about the selection were given. With less than $5 \%$ of stimuli the subject was unable to make a choice and that trial was rejected. Subjects received 400 trials, in eight sets of 50 stimuli. The orientation of the coil (that is, which surface was placed on the scalp) was changed randomly during the rest period (1-5 minutes) between each set of trials. All trials were preceded by 1-2 $\mathrm{s}$ with a verbal warning "stimulus now". In six subjects on a separate day a prolonged DC stimulus was used in an otherwise identical protocol. The direct current reached a peak of $0.2-0.4 \mathrm{~mA}$ within $1 \mathrm{~s}$ and was maintained for about 4-5 s. It was derived from a specially designed unit which has been used previously to document changes in complex reaction times. ${ }^{13}$ The current was usually applied via two standard silversilver chloride electrodes, fixed to the scalp with collodion at C3 and C4 and filled with conductive paste. The current was monitored. These stimuli were adjusted to be below threshold for a skin sensation.

\section{Results}

The principal results for right-handed subjects are summarised in the table. With the magnetic stimuli, there was a group preference for selecting the right hand in 1155 of the 1800 trials in which the current was clockwise and in 771 of 
Table Summary of results in group of right-handed subjects

\begin{tabular}{lcc}
\hline & \multicolumn{2}{c}{ hand selected } \\
\cline { 2 - 3 } Magnetic stimulus current & left & right \\
\hline $\begin{array}{l}\text { anticlockwise } \\
\text { clockwise }\end{array}$ & 1029 & 771 \\
9 subjects, $\mathrm{N}=3600, \chi^{2}=163 \cdot 8, \mathrm{p}<0.001$ & 1155 \\
DC-stimulus current & & \\
$\mathrm{C} 4$ positive & 638 & 562 \\
$\mathrm{C} 3$ positive & 623 & 577 \\
\hline subjects, $\mathrm{N}=2400, \chi^{2}=0.4, \mathrm{~ns}$ & & \\
\hline ns $=$ not significant. & &
\end{tabular}

the 1800 trials in which it was anticlockwise. This difference between hand selection (dependent upon current direction) was highly significant $(\mathrm{p}<0.001)$. A similar trend was evident in all subjects and in seven it was significant at the $5 \%$ level (fig). In contrast, as previously reported, ${ }^{14}$ the DC stimulator produced no preference for selection of a particular hand when electrodes were at $\mathrm{C} 3$ and C4 ( $p=0.50$, see the table) or F3 and F4. Subjects were apparently unaware that the transcranial magnetic stimuli influenced hand selection. When questioned they felt that their decisions appeared to be made in an entirely natural way. For results in left-handed subjects see below.

\section{Discussion}

The major implication from this study is that the neural events activated by a single transcranial stimulus can alter the selection of a particular motor programme or strategy (to move the left or the right hand) dependent upon the direction of the intracranial current. The stimuli were subthreshold for an evoked EMG response in the relaxed distal muscles so that the neural mechanism is not dependent upon production of a peripheral movement or, presumably, on activation of a significant number of motoneurons in upper or lower limb muscles. This finding would imply that cortical

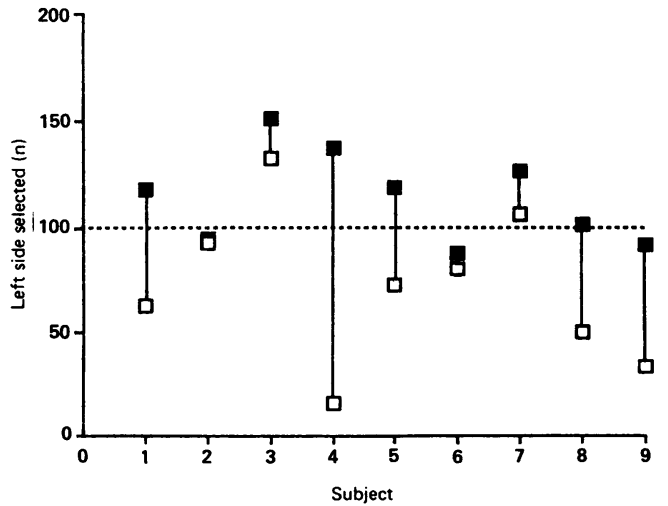

Figure Data from nine right-handed subjects shown on separate vertical lines. Single magnetic stimuli were delivered and then the subject was required to select and move either the left or right index finger (see text). The number of times the left hand was selected is shown for anti-clockwise (filled symbol) and clockwise currents (open symbol). Two hundred stimuli were delivered with each direction of current. In seven of the subjects there was a significant difference in the bias for hand selection according to the direction of induced current, with clockwise current associated with selection of the right hand. processing can be influenced by levels of magnetic stimulation which are "subthreshold" for a motor response (see below).

Interestingly the direction of transient current flow (clockwise) associated with preferred selection of the right hand was also the direction associated with a lower threshold for activation of the output from the left motor cortex to muscles of the right upper limb. ${ }^{89}$ However, these events are not necessarily linked given that we have observed the opposite effect on hand preference in three left-handed subjects. Thus, clockwise current led to an increased selection of the left hand $(280 / 500$ trials, $\mathrm{p}<0.01$ ) and anticlockwise current to increased selection of the right hand $(297 / 500$ trials, $\mathrm{p}<0.01$ ) and these trends were significantly different (Chi-Square-23.0 $\mathrm{p}<0.001$ ).

Furthermore, the current flow required to produce the preference in hand selection was not mimicked by magnetic stimulation with the coil positioned posterior to the vertex (near $\mathrm{Oz}$ ). This was demonstrated for a group of five right-handed subjects from the initial study. With a posterior position the probability of selection of the left and right hands was 0.504 and 0.496 , irrespective of the direction of occipital current. The stimulus intensity was again below movement threshold for the limbs. The exact cellular elements responsible for the change in hand preference are presumably anterior to, or can be influenced anterior to the motor cortex, and they may be organised symmetrically in those that are left- and righthanded. Presumably, areas upstream of the motor cortex participate in the phenomenon. While blinking and ocular movements may have occurred in some trials with the magnetic stimuli they are unlikely to have mediated the effects given the different behaviour of those that were left- and right-handed.

These data suggest that neural elements involved in motor planning can be activated with some selectivity by transcranial magnetic stimulation. Further studies are required to document the effects of transcranial stimulation on other aspects of motor planning and higher cortical functions.

Finally, until the full neural effects of single transcranial electrical and particularly magnetic stimuli are documented, this study emphasises that it remains prudent to restrict the numbers of stimuli to the minimum required for the clinical or experimental investigation.

This study was supported by the National Health and Medical Research Council. The authors are grateful to Professor $N$ Birbaumer (Tübingen, FRG) for provision of the DC stimulator used in his previous studies and to Professor D Burke for comments on the manuscript.

1 Merton PA, Morton HB. Stimulation of the cerebral cortex in the intact human subject. Nature 1980;285:227.

2 Barker AT, Jalinous R, Freeston IL. Non-invasive stimulation of human motor cortex. Lancet 1985;i:1106-7.

3 Rothwell JC, Thompson PD, Day BL, Dick JPR, Kachi T, Cowan JMA, Marsden CD. Motor cortex stimulation in intact man. 1. General characteristics of EMG responses intact man. 1. General characteristics of EMG

4 Gandevia SC, Rothwell JC. Activation of the human diaphragm from the motor cortex.J Physiol 1987;384:109-18. 
5 Day BL, Rothwell JC, Thompson PD, Dick JPR, Cowan JMA, Berardelli A, Marsden CD. Motor cortex stimulation in intact man. 2. Multiple descending volleys. Brain 1987;110:1191-209.

6 Hess CW, Mills KR, Murray NMF. Percutaneous stimulation of the human brain: a comparison of electrical and magnetic stimuli. J Physiol 1986;378:35.

7 Day BL, Dick JPR, Marsden CD, Thompson PD. Differences between electrical and magnetic stimulation of the human brain. J Physiol 1986;378:36.

8 Hess CW, Mills KR, Murray NMF. Responses in small hand muscles from magnetic stimulation of the human brain. J Physiol 1987;388:397-419.

9 Day BL, Dressler D, Maertens de Noordhout A, et al. Electric and magnetic stimulation of the human motor cortex: surface EMG and single motor unit responses. $J$ Physiol 1989;412:449-73.

10 Amassian VE, Cracco RQ, Maccabee PM. A sense of movement elicited in paralyzed distal arm by focal magnetic coil stimulation of human motor cortex. Brain Res 1989;479:355-60.

11 Day BL, Rothwell JC, Thompson PD, et al. Delay in the execution of voluntary movement by electrical or mag netic brain stimulation in intact man. Evidence for the storage of motor programs in the brain. Brain 1989;112:649-63.
12 Oldfield $R$. The assessment and analysis of handedness: The Edinburgh Inventory. Neuropsychologia 1971;9:97-113.

13 Lutzenberger $\mathbb{W}$, Elbert T. Assessment of the effects of weak electric currents on brain and behaviour. In: Schmidt A, ed. Safety Assessment of Clinical NMR Technique. ed. Safety Assessment

14 Jaeger D, Elbert $T$, Lutzenberger $W$, Birbaumer $N$. The effects of externally applied transcephalic weak direct currents on lateralisation in choice reaction tasks. Psychophysiol 1987;1:127-33.

\section{Erratum}

Since this study was conducted we have determined that the manufacturer's specification of the direction of current flow in the Novametrix magnetic stimulator was in error. The possibility of this problem was first brought to our notice by Dr B Day and colleagues (personal communication, June 1990), and Day and colleagues (personal communication, June 1990), and
has been found to apply to the stimulator used in this study. Thus throughout the text (and in the table) the word "anticlockwise" should be replaced by "clockwise" and vice versa.
Previous published studies using this device may also contain this error. 